\title{
Sexual Dysfunction and its Determinants in Male Patients with Rheumatoid Arthritis
}

\author{
Hassan O. Ghareeb ${ }^{1}$ D , Ghada M. Khafagy ${ }^{2}$ D , Hatem H. Eleishi ${ }^{3}$, Hala A. Hussein ${ }^{2 \star}$, Marwa D. Hasan ${ }^{2}$ \\ ${ }^{1}$ Department of Obstetrics and Gynecology, Faculty of Medicine, Cairo University, Cairo, Egypt; ${ }^{2}$ Department of Family Medicine, \\ Faculty of Medicine, Cairo University, Cairo, Egypt; ${ }^{3}$ Department of Rheumatology, Faculty of Medicine, Cairo University, Cairo, \\ Egypt
}

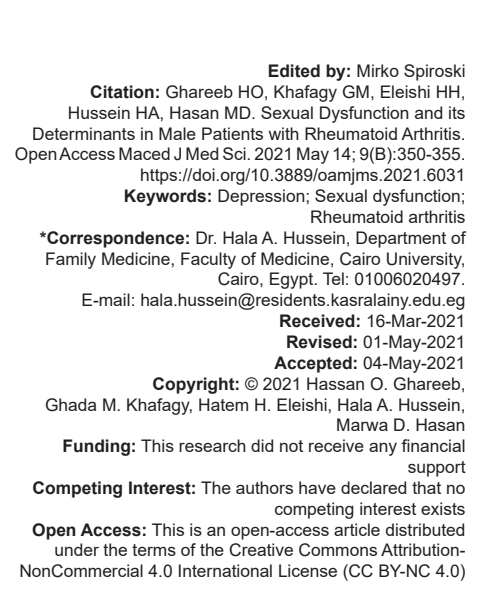

\section{Introduction}

Rheumatoid arthritis (RA) is a chronic systemic autoimmune disease that mainly affects joints and results in warm, swollen, painful, and stiff joints that often worsen after rest and may also affect other parts of the body in the form of low red blood cell count, inflammation around the lungs, and inflammation around the heart [1].

$\mathrm{RA}$ is a major global public health challenge. The age-standardized prevalence and incidence rates are increasing [2]. This is between 0.5 and $1 \%$ of adults in the developed world with 5 and 50 per 100,000 people newly developing the condition each year [3].

Sexuality is a major aspect of humans' quality of life as it is responsible for our individual welfare. Sexual dysfunction can be defined as a shift in any aspect of sexual activity that can lead to discomfort, pain, and reduced sexual intercourse. Chronic diseases such as RA are considered to affect the quality of sexual life [4]. Compared to the general population, sexual health and sexual activity are often significantly affected in patients with RA [5].

Sexual disorders may be experienced in RA because of physical causes such as discomfort, morning stiffness, reduced joint movements, and inability to perform satisfactory physical tasks, mental and/or psychological issues such as depression, apprehension, and concerns about disfigured body image, concerns from fear of unattractiveness, and many other reasons such as increased disease activity, poor functional status, and decreased libido [6].

One of the most important issues about the treatment of sexual dysfunction associated with RA is the fact that neither is sexual functioning routinely addressed by physicians or health professionals [7], nor are sexual problems freely raised by males due to our culture and tradition constraints.

Thus, we conducted our study to go deep into this extremely sensitive issue to determine the possible different risk factors for sexual dysfunction in male 
patients with RA and to study the magnitude of the problem among RA patients.

\section{Subjects and Methods}

\section{Study setting}

The study was conducted in the Rheumatology and Family Medicine Outpatient Clinics, in Kasr Alainy Faculty of Medicine, Cairo University, from January 2019 to May 2020.

There are two rheumatology outpatient clinics in Kasr Alainy Hospital: One for new cases and the other for follow-up from which we selected our participants.

\section{Study design}

This study is a case-control study carried on a purposive sample, based on the previous study of Nasr and El-Shafey [8]. This revealed that the percentage of sexual dysfunction among the control group was $11 \%$ versus $46 \%$ in the RA patients using an open API program (power 90\%, Cl 95\%). Accordingly, the minimum required sample was found to be 60 (30 in each group).

Every adult married participant above 18 years of age, meeting the inclusion criteria of the study, diagnosed based on the 2010 American College of Rheumatology/European League Against Rheumatism RA classification criteria, and attending the rheumatology clinic was selected, and any normal participants in the same age group and fitting the same criteria from either the relatives of the included patients or from patients complaining from non-related problems represent the control group.

Ethical approval was obtained from the Research Committee in the Faculty of Medicine, Cairo University (IRB: N23-2019). Informed written consent was taken from all patients after full explanation of the purpose of the study and benefits and ensuring confidentiality of the collected data.

We excluded males who were widowed or divorced, those who smoked or drank alcohol, those who had any other previous known medical problems, for example, hypertension and thyroid, before the diagnosis of RA, and those taking medications that affect sexual function, for instance, androgens, anticonvulsants, barbiturates, or antidepressants or those who have been on corticosteroid therapy for more than 6 months at the time of the study.

The case group was subjected to the assessment of sociodemographic data (A El-Gilany) [9] and medical assessment regarding duration of the disease, complications, and drugs currently used and regarding sexual function using the international index of erectile function (IIEF) questionnaire (RC Rosen) [10] investigating the following items: Erectile dysfunction (ED), sexual desire (libido), orgasmic problems, ejaculatory problems, and sexual intercourse frequency. The Arabic version of the questionnaire seemed to be reliable and valid, with a high degree of specificity (R Shamloul) [11].

The disease activity score in 28 joints (DAS-28) is a rating system that is commonly used in clinical practice to determine treatment efficacy and track RA patients' disease activity (Favalli et al.) [12]. Disability caused by the disease was determined using the health assessment questionnaire (HAQ) which evaluates patient difficulties with daily living activities over the past week (Carreno et al.) [13] and has an Arabic version which is valid and reliable in assessing disability in Arabic-speaking countries (El Miedany et al.) [14]. Assessment of depression was carried out using the Arabic version of the patient health questionnaire (PHQ-9) which is valid and reliable in assessing depression in Arabic-speaking countries (AlHadi et al.) [15]. A morning blood sample of $5 \mathrm{ml}$ was obtained from all males from both groups. The serum total testosterone level was determined after electrochemiluminescence equipment prepared and centrifuged the blood samples.

The control group was subjected to the assessment of sociodemographic data, sexual function using the IIEF, depression using $\mathrm{PHQ}-9$, and serum hormonal level.

\section{Statistical data analysis}

All data were collected, tabulated, and statistically analyzed using SPSS 26.0 for Windows (SPSS Inc., Chicago, IL, USA).

Quantitative data were presented as the mean \pm SD and median (range), and qualitative data were presented as absolute frequencies (number) and relative frequencies (percentage).

Independent samples Student's t-test was used to compare between two groups of normally distributed variables. Percentages of categorical variables were compared using the Chi-square test or Fisher's exact test when appropriate.

Pearson's rank correlation coefficient was calculated to assess the relationship between various study variables. A + sign indicates a direct correlation and a - sign indicates an inverse correlation. Furthermore, values near 1 indicate a strong correlation and values near 0 indicate a weak correlation. $p<0.05$ was considered statistically significant (S); $p \geq 0.05$ was considered statistically insignificant (NS). 


\section{Results}

\section{Section 1: Sociodemographic data of both groups and clinical characteristics of the case group}

Both groups were matched with no statistically significant difference regarding age, education, occupation, social class, and BMI ( $p>0.05)$.

Most of the cases (60\%) had mild impact on quality of life. The most reported comorbidity in cases was diabetes (3.3\%). The most reported complication in cases was subcutaneous nodules (13.3\%).

\section{Section 2: Comparison between the two} groups regarding PHQ-9 score, sexual function score, and serum testosterone level

Table 1 shows that there was a statistically significant difference between the groups regarding depression scores $(p<0.05)$. The mean PHQ score was $11.86 \pm 7.84$ among the case group versus $7.36 \pm$ 5.99 among the control, there was a highly statistically significant difference between both groups regarding all domains of sexual functions as well as the total score of the IEEF (mean score $42.73 \pm 25.65$ in the case group vs. $61.2 \pm 14.41$ in the control group), and there was a statistically significant difference between both groups regarding free and total testosterone levels $(p<0.05)$.

Table 1: Comparison between the case and control groups regarding sexual function domains, PHQ results, and serum testosterone levels

\begin{tabular}{llll}
\hline Item & Case $(n=30)$ & Control $(n=30)$ & $p$-value \\
\hline Sexual function domains (mean \pm SD) & & \\
$\quad$ Erection domain & $17.70 \pm 10.96$ & $24.30 \pm 6.02$ & 0.005 \\
Intercourse domain & $7.06 \pm 5.15$ & $11.20 \pm 3.06$ & $<0.001$ \\
Orgasm domain & $5.76 \pm 4.24$ & $8.80 \pm 2.10$ & 0.001 \\
$\quad$ Desire domain & $5.93 \pm 2.83$ & $8.36 \pm 1.88$ & $<0.001$ \\
$\quad$ Satisfaction domain & $6.26 \pm 3.09$ & $8.53 \pm 3.09$ & 0.002 \\
$\quad$ Total score IEEF & $42.73 \pm 25.65$ & $61.20 \pm 14.41$ & 0.001 \\
Total testosterone (N \& \%) & & & \\
$\quad$ Low & $13(43.3 \%)$ & $2(6.7 \%)$ & 0.001 \\
$\quad$ Normal & $17(56.7 \%)$ & $28(93.3 \%)$ & \\
Free testosterone & $9(30 \%)$ & $2(6.7 \%)$ & 0.020 \\
$\quad$ Low & $21(70 \%)$ & $28(93.3 \%)$ & \\
$\quad$ Normal & $11.86 \pm 7.84$ & $7.36 \pm 5.99$ & 0.006 \\
PHQ severity (mean $\pm \mathrm{SD})$ & &
\end{tabular}

\section{Section 3: Relation between sexual} dysfunction and severity of depression, disease severity (DAS), and impact on daily life activities (HAQ)

Table 2 shows that there was a highly statistically significant relation between depression grades and sexual dysfunction with most cases with moderate-to-severe depression complaining of sexual dysfunction $(p<0.001)$ (OR of sexual dysfunction among depressed patients 11.2 [0.7-72.3]). There was a highly statistically significant relation between DAS grades and sexual dysfunction with most cases with moderate-to-severe arthritis complaining of sexual dysfunction $(p<0.001)$. Furthermore, there was a highly statistically significant relation between $\mathrm{HAQ}$ severity and sexual dysfunction with most cases with moderate and severe impact on quality of life complaining of sexual dysfunction $(p<0.001)$.

Table 2: Relation between sexual dysfunction and depression, disease severity, and disease effect on daily life activities among RA patients

\begin{tabular}{llll}
\hline Degree & ED grade & p-value \\
\cline { 2 - 3 } & With dysfunction $(n=18)$ & Without dysfunction $(n=12)$ & \\
\hline DAS score & & 5 & $<0.001$ \\
$\quad$ Non $(n=2)$ & 0 & 5 & \\
Mild $(n=7)$ & 2 & 2 & \\
Moderate $(n=6)$ & 4 & 0 & $<0.001$ \\
Severe $(n=12)$ & 12 & & \\
HAQ severity & & 12 & \\
Mild $(n=18)$ & 6 & 0 & 0.006 \\
Moderate $(n=9)$ & 9 & 0 & \\
Severe $(n=3)$ & 3 & & \\
PHQ score & 16 & 5 & \\
Yes & 2 & 7 & \\
No & & &
\end{tabular}

\section{Section 4: Relation between serum} testosterone levels and sexual dysfunction grade in the groups

Table 3 shows that there was a statistically significant relation between ED and free and total testosterone levels with more sexual dysfunction being associated with a lower hormone level $(p<0.05)$.

Table 3: Relation between total and free testosterone results and ED grade between both groups

\begin{tabular}{llll}
\hline Degree & ED & p-value \\
\cline { 2 - 3 } & With dysfunction $(n=36)$ & Without dysfunction $(n=24)$ & \\
\hline Total testosterone & 12 & 1 & $0.002^{* *}$ \\
$\quad$ Abnormal & 6 & 11 & $0.034^{* *}$ \\
$\quad$ Normal & 6 & 1 & \\
$\begin{array}{l}\text { Free testosterone } \\
\text { Low }\end{array}$ & 8 & 11 & \\
$\quad 10$ & & &
\end{tabular}

\section{Section 5: Correlations between total} sexual dysfunction score and different diseaserelated parameters

Table 4 shows that there was a statistically significant negative correlation between $\mathrm{HAQ}$ score, $\mathrm{PHQ}$ scores, RA duration, and sexual function with increased disease impact on quality of life and increased depression being associated with decreased sexual function. There was a statistically significant positive correlation between testosterone levels and sexual function with increased testosterone levels associated with better sexual function.

Table 4: Correlation between total ED score and different parameters

\begin{tabular}{lll}
\hline Parameters & \multicolumn{2}{l}{ Total sexual function } \\
\cline { 2 - 3 } & $\mathrm{R}$ & $\mathrm{p}$-value \\
\hline HAQ score & -0.854 & $0.000^{\star *}$ \\
PHQ score & -0.789 & $0.000^{\star *}$ \\
RA duration & -0.592 & $0.001^{*}$ \\
Total testosterone & 0.583 & $0.001^{*}$ \\
Free testosterone & 0.514 & $0.004^{*}$ \\
\hline
\end{tabular}




\section{Discussion}

Our study revealed that sexual function was greatly affected in RA patients compared to healthy controls and that RA patients also suffer from depression more than healthy people.

Sexual function was significantly affected by disease activity, physical disability, and serum hormonal level.

Our study found that there was a highly statistically significant difference between both male groups regarding all domains of sexual function as well as the total score of the IEEF $(p<0.05)$ with RA male patients having lower sexual function.

This dysfunction could be due to persistent pain, frequent disease activity, physical disability and dependence on others, joint stiffness, fatigue, psychological status, and/or treatment with certain drugs.

Inadditiontothat, elevatedlevelsofinflammatory biomarkers, for example, C-reactive protein, tumor necrosis factor-alpha, and some interleukins (IL-6 and IL-18), are associated with increased risk of vascular ischemic events and atherosclerotic changes [16].

IL-6 has been shown to affect vascular disease, lipid metabolism, mitochondrial activities, the neuroendocrine system, and neuropsychological behavior [17].

These results are confirmed by the study of Alia et al. [18] which included 50 men (37 spondylarthritis $[\mathrm{SpA}]$ and $13 \mathrm{RA}$ ) patients and 50 healthy, age-matched controls to assess the effect of rheumatic diseases on sexual performance. Sexual function of patients and controls was evaluated by the IIEF and it was found that patients with $\mathrm{SpA}$ or RA have more severe ED in comparison with healthy controls.

These results are also in agreement with the study of El Miedany et al. [14] which showed that sexual dysfunction is prevalent among RA males in comparison with controls and ED was significantly correlated $(p<0.01)$ with pain score and disease activity.

Our study reported a highly statistically significant difference between both groups regarding depression, as well as a highly statistically significant relation between depression and sexual dysfunction with the majority of RA cases with moderate-to-severe depression complaining of sexual dysfunction $(p<0.05)$.

Living with pain, stiffness, fatigue, limited movement, and decreased strength associated with arthritis may reduce ability of sexual expression and enjoyment. In addition to that, arthritis results in restriction of normal activities and might distort selfimage, self-perception, and self-esteem resulting in depression and thus reluctance to engage in a physical relationship.
Both clinical studies and recent meta-analyses have indicated that IL- 6 is the most consistently elevated cytokine in the blood of patients with major depressive disorder [19].

Although the mechanism by which cytokines cause depression is not fully understood, it may be through hyperactivation of the HPA axis which is associated with psychopathologies such as depression, panic disorder, and anxiety [20].

These results are also in accordance with a systematic review that included a total of 72 studies, carried out on 13,189 patients who were eligible for inclusion in the review, and revealed that the prevalence of major depression according to the PHQ-9 was $38.8 \%(95 \% \mathrm{Cl} 34 \%, 43 \%)$ and so depression is highly prevalent in RA and is associated with poorer RA outcomes. This suggests that optimal care of RA patients should include detection and management of depression [21].

There was a highly statistically significant relation between DAS grades and sexual problems with most cases with moderate-to-severe arthritis complaining of sexual problems $(p<0.001)$. This could be explained by the impairment of physical fitness in RA patients as loss of muscle mass and muscle strength is common in RA patients and is proportional to disease activity. This loss of muscle strength is associated with a higher grade of disability and a poorer quality of life.

Sexual activity is considered a type of physical exercise with energy expenditure that is classified as moderate to vigorous physical activity. Therefore, a certain level of physical fitness is required, which is often limited by the disease in RA patients.

This loss of muscle mass and muscle strength in RA patients is a consequence of chronic inflammation and the catabolic effect of inflammatory cytokines which directly affect sexual function [22].

In addition, low testosterone levels as a consequence of chronic inflammation might be important since this leads to loss of muscle mass and strength as well as to a decline in sexual function.

These results agreed with the study of Gaber et al. [23] which included 216 RA patients and 187 matched healthy controls. This study revealed that RA patients, either male or female, complained of sexual dysfunction with all sexual dysfunction parameters being significantly higher in RA patients than in the controls. The study revealed also that sexual dysfunction was associated with disease severity as active RA patients were associated with a higher risk of sexual dysfunction (OR 2.7) $(p=0.03)$ and OR increased to 5.6 in patients with severe disease activity.

The current study revealed that there was a statistically significant difference between both male groups regarding free and total testosterone levels $(p<0.05)$ with lower testosterone levels in RA patients. 
Furthermore, there was a statistically significant relation between ED and free and total testosterone levels $(p<0.05)$. There was a statistically significant positive correlation between testosterone levels and sexual function with increased testosterone levels associated with better sexual function.

This may be explained by the anti-inflammatory effects of gonadal and adrenal androgens in RA. Hypoandrogenism was found to be associated with RA. The level of testosterone reduces at the onset of disease complications and returns to the normal state after relieving the acute symptoms. The severity of gonadotropin suppression is associated with the severity of disease [24].

The proven fact is that there is an association between low testosterone levels and RA. Some authors believe that it is due to the disease process itself and a consequence of the disease rather than a cause as, in early RA, current inflammation seemed to affect the hypophyseal gonadal axis, mainly at the gonadal rather than the hypothalamic-pituitary level [25]. On the other hand, some authors thought that low testosterone might be a risk factor for later development of RA [26].

These results are consistent with the study of Lashkari et al. in 2018 [26] that were conducted on 59 patients with RA and 61 healthy gender- and agematched controls. Serum-free testosterone and DHEA levels were measured and compared between the studied groups. RA patients reported significantly lower testosterone and DHEA levels than the control group.

The results of the present study agreed with the study of Nasr and El-Shafey [8] which were conducted to compare serum androgen levels in male patients with RA and healthy males and to compare the presence of EDs between both groups. The study included 24 male patients with RA and 18 healthy controls. The study illustrated that there were highly statistically significant differences in the levels of serum DHEA and total and free testosterone levels between patients and controls ( $p<0.001)$. In addition, EDs were more frequent in RA patients (45.8\%) than in the control subjects $(11.1 \%)$. There were highly statistically significant correlations between total and free testosterone levels and the sexual dysfunction score $(p<0.001)$ which means increased sexual satisfaction with increased levels of testosterone.

The present study results are also in accordance with a case-control study conducted in Sweden. The study reported that the level of serum testosterone in patients with RA was significantly lower than in the control group and an abnormal serum testosterone level was significantly lower in patients with RA and positive rheumatoid factor. This means that hormonal changes are associated with RA and can affect the phenotype of the disease [27].

Our study has some limitations. For example, although our sample size is calculated statistically, it would be better to conduct the study on a larger sample from different age groups and different socioeconomic levels. In addition to that, a few participants were reluctant about going deep into their sexual issues.

To the best of our knowledge, this study is one of the few studies done discussing sexual problems among RA patients.

\section{Conclusion}

Sexual dysfunction is a prominent problem in male patients suffering from RA and it greatly affects the quality of life. The most prominent risk factors for sexual dysfunction in RA patients are depression, disease activity, disease-related disability, and serum testosterone levels.

\section{Implications for practice and/or research}

Based on our study findings, patients with RA greatly suffer in different aspects and their quality of life is reduced in many forms. More care must be directed to those patients to enhance their quality of life and satisfaction and we recommend assessment of sexual function of RA males as a part of their routine assessment of disease status. More prospective studies are needed on large scales to identify their main distressing issues and support them.

\section{References}

1. Kumar M. Clinical evaluation of patients suffering from rheumatoid arthritis with respect to iron deficiency. Int J Med Biomed Stud. 2019;3(7):178-2.

2. Safiri S, Kolahi AA, Hoy D, Smith E, Bettampadi D, Mansournia MA, et al. Global, regional and national burden of rheumatoid arthritis 1990-2017: A systematic analysis of the global burden of disease study 2017. Ann Rheum Dis. 2019;78(11):1463-71. https://doi.org/10.1136/ annrheumdis-2019-215920

PMid:31511227

3. Smolen JS, Burmester GR, Combeet B; NCD Risk Factor Collaboration (NCD-RisC). Worldwide trends in diabetes since 1980: A pooled analysis of 751 population-based studies with 4.4 million participants. Lancet. 2016;387(10027):1513-30. https://doi.org/10.7554/elife.13410.019

PMid:27061677

4. Almeida PH, Ferreira CD, Kurizky PS, Muniz LF, Mota LM. How the rheumatologist can guide the patient with rheumatoid arthritis on sexual function. Rev Bras Reumatol. 2015;55(5):458-63. https://doi.org/10.1016/j.rbre.2014.08.008 PMid:25794992

5. Khnaba D, Rostom S, Lahlou R, Bahiri R, Abouqal R, HajjajHassouni N. Sexual dysfunction and its determinants in 
Moroccan women with rheumatoid arthritis. Pan Afr Med J 2016;24:16. http://org.doi.10.11604/pamj.2016.24.16.9081.

6. Josefsson KA, Gard G. Sexual health in patients with rheumatoid arthritis: Experiences, needs and communication with health care Wiley online library. Musculoskelet Care. 2012;10(2):76-89. https://doi.org/10.1002/msc.1002

PMid:22223288

7. Tristano AG. Impact of rheumatoid arthritis on sexual function. World J Orthop. 2014;5(2):107-11.

PMid:24829873

8. Nasr MM, El-Shafey AM. Sexual performance in rheumatoid arthritis patients an unnoticed problem. Egypt Rheumatol. 2013;35(4):201-5. https://doi.org/10.1016/j.ejr.2013.07.001

9. El-Gilany A, El-Wehady A, El-Wasify M. Updating and validation of the socioeconomic status scale for health research in Egypt. East Mediterr Health J. 2012;18(9):962-8. https://doi. org/10.26719/2012.18.9.962 PMid:23057390

10. Rosen RC, Riley A, Wagner G, Osterloh IH, Kirkpatrick J, Mishra A. The international index of erectile function (IIEF): A multidimensional scale for assessment of erectile dysfunction. Urology. 1997;49(6):822-30. https://doi.org/10.1016/ s0090-4295(97)00238-0

\section{PMid:9187685}

11. Shamloul R, Ghanem H, Abou-zeid A. Validity of the Arabic version of the sexual health inventory for men among Egyptians. Int J Impot Res. 2004;16(5):452-5. https://doi.org/10.1038/ sj.ijir.3901248

PMid:15175638

12. Favalli EG, Becciolini $A$, Biggioggero $M$, Marchesoni $A$, MeroniPL. Is there a need for new thresholds to define remission and low disease activity by disease activity score 28 calculated with $\mathrm{C}$ reactive protein? Real life data from a localregistry. Ann Rheum Dis. 2015;74(1):e5. https://doi.org/10.1136/ annrheumdis-2014-206651 PMid:25304319

13. Carreno A, Fernandez I, Badia X, Varela C, Roset M. Using HAQ-DI to estimate HUI-3 and EQ-5D utility values for patients with rheumatoid arthritis in Spain. Value Health. 2011;14(1):192200. https://doi.org/10.1016/j.jval.2010.11.001 PMid:21211502

14. El Miedany Y, El Gaafary M, El Aroussy N, Youssef S, Ahmed I. Sexual dysfunction in rheumatoid arthritis patients: Arthritis and beyond. Clinical Rheumatol. 2012;31(4):601-6. https://doi. org/10.1007/s10067-011-1891-2 PMid:22108779

15. AlHadi AN, AlAteeq DA, Al-Sharif E, Bawazeer HM, Alanazi H, AIShomrani AT, et al. An Arabic translation, reliability, and validation of patient health questionnaire in a Saudi sample. Ann Gen Psychiatry. 2017;16:32. https://doi.org/10.1186/ s12991-017-0155-1

PMid:28878812

16. Emerging Risk Factors Collaboration; Kaptoge $S, \quad D i$ Angelantonio E, Lowe G, Pepys MB, Thompson SG, Collins R, et al. C-reactive protein concentration and risk of coronary heart disease, stroke, and mortality: An individual participant meta-analysis. Lancet. 2010;375(9709):132-40. https://doi. org/10.1016/s0140-6736(09)61717-7

17. Hunter CA, Jones SA. IL- 6 as a keystone cytokine in health and disease. Nat Immunol. 2015;16(5):448-57.

PMid:25898198

18. Alia F, Leila R, Khereddine D, Miladi S, Kmar O, Leila S, et al Comparison of sexual function in men with spondyloarthritis and rheumatoid arthritis. Egypt Rheumatol. 2020;42(4):275-9.

19. Haapakoski R, Mathieu J, Ebmeier KP, Alenius H, Kivimäki M. Cumulative meta-analysis of interleukins 6 and $1 \beta$, tumour necrosis factor $\alpha$ and C-reactive protein in patients with major depressive disorder. Brain Behav Immun. 2015;49:206-15. https://doi.org/10.1016/j.bbi.2015.06.001 PMid:26065825

20. Du X, Pang TY. Is dysregulation of the HPA-axis a core pathophysiology mediating co-morbid depression in neurodegenerative diseases? Front Psychiatry. 2015;6:32. https://doi.org/10.3389/fpsyt.2015.00032

PMid:25806005

21. Matcham F, Rayner L, Steer S, Hotopf M. The prevalence of depression in rheumatoid arthritis: A systematic review and meta-analysis. Rheumatology (Oxford) 2013;52(12):2136-48. https://doi.org/10.1093/rheumatology/ket169

PMid:24003249

22. Dorner TE, Berner C, Haider S, Grabovac I, Lamprecht T, Fenzl KH, et al. Sexual health in patients with rheumatoid arthritis and the association between physical fitness and sexual function: A cross-sectional study. Rheumatol Int. 2018;38(6):1103-14. https://doi.org/10.1007/s00296-018-4023-3 PMid:29644435

23. Gaber W, Moghazy A, Niazy M, Salem HK. Risk factors for sexual dysfunction in Egyptian patients with rheumatoid arthritis and its relation to disease activity. Egypt Rheumatol. 2017;39(3):135-8. https://doi.org/10.1016/j.ejr.2017.01.001

24. da Silva JA. Relationships between glucocorticoids and gonadal steroids in rheumatoid arthritis. Ann N Y Acad Sci. 2002;966:158-65.

PMid:12114269

25. Karlson EW, Chibnik LB, McGrath M, Chang SC, Keenan BT, Costenbader $\mathrm{KH}$, et al. A prospective study of androgen levels, hormone-related genes and risk of rheumatoid arthritis. Arthritis Res Ther. 2009;11(3):R97. https://doi.org/10.1186/ar2742 PMid:19555469

26. Lashkari M, Noori A, Oveisi S, Kheirkhah M. Association of serum testosterone and dehydroepiandrosterone sulfate with rheumatoid arthritis: A case control study. Electron Physician. 2018;10(3):6500-5. https://doi.org/10.19082/6500 PMid:29765575

27. Pikwer M, Giwercman A, Bergström U, Nilsson JA, Jacobsson LT, Turesson C. Association between testosterone levels and risk of future rheumatoid arthritis in men: A population-based casecontrol study. Ann Rheum Dis. 2014;73(3):573-9. https://doi. org/10.1136/annrheumdis-2012-202781

PMid:23553100 